\title{
Descrição do desenvolvimento neuropsicomotor e visual de crianças com deficiência visual
}

\author{
Description of the neuropsychomotor and visual development of visually impaired children
}

Telma de Araujo Souza ${ }^{1}$, Vivian Estevam de Souza ${ }^{2}$, Marcia Caires Bestilleiro Lopes ${ }^{3}$, Silvia Prado Smit Kitadal ${ }^{4}$

\section{RESUMO}

Objetivo: Avaliar o desenvolvimento neuropsicomotor e visual de crianças com deficiência visual.

Métodos: Foram avaliadas 45 crianças, de ambos os gêneros, em um período de 6 meses. As crianças foram distribuidas em dois grupos de estudo: experimental e de controle. Dessa forma aplicou-se a avaliação do desenvolvimento neuropsicomotor e da funcionalidade visual.

Resultado: No grupo controle $86,66 \%$ da amostra estava inadequado quanto ao comportamento da coordenação, entretanto, todos os aspectos analisados estavam inadequados. As diferenças entre os grupos mostraram-se significativas, já que valores como $\mathrm{p}<0,05$ foram evidenciados, tanto no comportamento da funcionalidade visual, quanto no desenvolvimento neuropsicomotor. Conclusão: Na amostra estudada, observou-se que os deficientes visuais caracterizavam-se por apresentar atraso global, do desenvolvimento, principalmente no comportamento da coordenação.

Descritores: Desenvolvimento infantil; Acuidade visual; Transtornos psicomotores; Doenças do sistema nervoso

\section{ABSTRACT}

Purpose: To assess the neuropsychomotor and visual development of visually impaired children.

Methods: Fourty-five children of both genders were evaluated in a 6-months period. The children were distributed into two study groups: experimental and control. The neuropsychomotor development and visual efficiency were evaluated in the two groups.

Results: In the control group, $86.66 \%$ of the sample was inappropriate on the coordination behavior, however, all aspects were considered inadequate. The differences among the groups were significant, since $p$ values $<0.05$ were evidenced in the visual efficiency behavior as well as in the neuropsychomotor development.

Conclusion: It was observed in the studied sample that the visually impaired were characterized by a global delay in the neuropsychomotor development, mostly in the coordination behavior.

Keywords: Child development; Visual acuity; Psychomotor disorders; Nervous system diseases

\section{INTRODUÇÃO}

A integridade do desenvolvimento neuropsicomotor (DNPM) depende da organização dos sentidos pelo sistema nervoso central, sendo este a base para potencializar o desenvolvimento das habilidades humanas, do comportamento e da aprendizagem ${ }^{(1-4)}$. O DNPM realiza-se pelo prazer da criança em experimentar algo novo, como uma aquisição motora ou sensorial. A deficiência visual infantil pode então propiciar eventuais atrasos nesse tipo de desenvolvimento(5).

No processo de desenvolvimento infantil normal, a visão promove a integração das atividades motoras, perceptivas e mentais, ou seja, estabelece uma relação na qual a diminuição das capacidades vitais leva a uma série de comprometimentos em outras áreas do comportamento da criança, causados geralmente pela privação de certos estímulos. Sendo assim, no decorrer dos primeiros anos de vida a integração, a sintetização e a

Trabalho realizado no Departamento de Reabilitação Visual, Universidade de Santo Amaro UNISA - São Paulo (SP), Brasil.

${ }^{1}$ Fisioterapeuta, Ambulatório de Estimulação Visual Precoce, Universidade Federal de São Paulo - UNIFESP - São Paulo (SP), Brasil.

2 Fisioterapeuta, Universidade Federal de São Paulo - UNIFESP - São Paulo (SP), Brasil.

3 Fisioterapeuta, Ambulatório de Estimulação Visual Precoce, Universidade Federal de São

Paulo - UNIFESP - São Paulo (SP), Brasil; Universidade de Santo Amaro - UNISA - São Paulo (SP), Brasil.

${ }^{4}$ Médica, Universidade de Santo Amaro - UNISA - São Paulo (SP), Brasil.

Endereço para correspondência: Telma da Araújo Souza. Rua Mairipotaba, 52 - São Paulo (SP) CEP 02977-060 - E-mail: telmadeas@gmail.com

Recebido para publicação em 09.08.2009

Última versão recebida em 26.10.2010

Aprovação em 03.11.2010 interpretação das informações fornecidas por outros canais perceptivos devem ser amplamente exploradas nestas crianças ${ }^{(6-7)}$

A representatividade da deficiência visual permite considerar que, todos os anos, cerca de 500.000 crianças menores de 16 anos de idade ficam cegas no mundo, sendo que aproximadamente $90 \%$ delas residem em países em desenvolvimento ${ }^{(8-9)}$

A cegueira infantil representa 3,9\% dos casos de cegueira no mundo(5,10-11)

Em função das necessidades destas crianças, os profissionais da área de reabilitação têm procurado desenvolver aspectos conceituais que as caracterizem, orientando-as para o treinamento e a intervenção precoce de acordo com os princípios da medicina de reabilitação e de habilitação(12-13). Por parte dos fisioterapeutas, faz-se necessária a compreensão das características intrínsecas deste grupo para a efetiva intervenção, já que o déficit de integração sensorial pode comprometer o desenvolvimento ${ }^{(14-16)}$. O presente estudo foi desenvolvido tendo por objetivo avaliar o DNPM e também o desenvolvimento visual de crianças com deficiência, já que pesquisas específicas sobre estes aspectos ainda são escassas.

\section{MÉTODOS}

O presente estudo observacional, de corte transversal, realizou-se após a aprovação do comitê de ética da Universidade de Santo Amaro, em 2007, sob o número 104/2007. Foi desenvolvido no Ambulatório de Habilitação e Reabilitação Visual por um período de seis meses seguindo os princípios da Declaração de Helsinque. 
Foram avaliadas individualmente 45 crianças com idade entre 0 e 36 meses (média total de 22,9 meses) de ambos os sexos, cujos pais/responsáveis/cuidadores consentiram na utilização dos dados por meio da assinatura do termo de consentimento livre e esclarecido. Os participantes foram submetidos a duas avaliações: de DNPM, por meio da tabela elaborada com base na literatura de Arnold Gesell (Anexo 1)(7), composta por quatro escalas comportamentais: motora, de coordenação, de linguagem e social; e de visão funcional, utilizando-se a tabela da American Foundation for the Blind (AFB) ${ }^{(17)}$. Para a aplicação das avaliações foi necessária aproximadamente 1 hora. Os participantes foram distribuídos em dois grupos (experimental e de controle) conforme os critérios de inclusão: crianças sem doenças sistêmicas associadas e sem diagnóstico médico de atraso do DNPM, sendo que no grupo experimental as crianças deveriam apresentar deficiência visual bilateral diagnosticada. Todas as crianças avaliadas permaneceram no estudo. De acordo com os critérios adotados, o grupo controle constituiu-se de 30 participantes (com média de 24,1 meses), enquanto o grupo experimental apresentou apenas 15 crianças (com média de 21,7 meses), sendo que os integrantes deste já realizavam estimulação visual precoce por um período médio de 8,6 horas. Os materiais utilizados e as atividades realizadas foram específicos e baseados na idade cronológica referenciada nas tabelas.

Os resultados obtidos ao término da avaliação permitiram considerar se os comportamentos das crianças estavam adequados ou não à sua faixa etária. Caso estivessem inadequados, seria necessário continuar a avaliação voltando às fases anteriores, a fim de se obter a idade comportamental apropriada. Na análise dos resultados todos os comportamentos foram considerados.

Para a análise da representatividade estatística, os dados deste estudo foram analisados através do teste t de Student e da Análise de Variância (ANOVA), sendo que os valores considerados significativos apresentaram nível de significância de $95 \%(p<0,05)$.

\section{RESULTADOS}

Os valores absolutos obtidos na análise do grupo experimental permitem considerar que o desempenho da coordenação apresentou-se atrasado, ou seja, inadequado quando comparado aos outros, fato referente ao número da amostra totalizando 13 crianças (86,66\%). Na tabela 1 nota-se o atraso do DNPM do grupo experimental em todos os comportamentos analisados, em comparação com o grupo controle. Os valores obtidos no comportamento social mostraram-se mais elevados, porém com relevância significativa $(p=0,04)$. Na avaliação da visão funcional tabela 2 notou-se novamente $p<0,05$.

A comparação entre os diferentes comportamentos advindos do Teste de Análise de Variância (ANOVA) não evidenciou diferença estatística significativa entre os comportamentos de um mesmo grupo (experimental), sendo $p>0,05$; em contrapartida, as diferenças apareceram quando houve comparação com a média de comportamento do grupo controle $(p<0,05)$, sendo os dados descritos na tabela 3.

Um dos achados da pesquisa, não relacionado com o objetivo principal, demonstra que a catarata congênita é o acometimento ocular mais frequentemente encontrado no grupo experimental, totalizando 9 crianças, fato também referenciado na literatura ${ }^{(18-21)}$.

\section{DISCUSSÃO}

Os transtornos de integração sensorial causam alterações no desenvolvimento em diversas áreas comportamentais (motoras, linguísticas, cognitivas e sociais) ${ }^{(22-23)}$. Com base nos dados coletados neste estudo, comprova-se que as crianças com deficiência visual apresentam atraso no desenvolvimento neuropsicomotor e na visão funcional em comparação com as crianças sem comprometimento, o que também foi confirmado no estudo desenvolvido por Cioni et al.(24).

Os resultados mostraram que o desempenho da coordenação foi o mais comprometido no grupo experimental, o que está de acordo com os achados das pesquisas relacionadas às manifestações que ocorrem nos primeiros anos de vida e que são pobremente experimentadas ${ }^{(24-25)}$. A deficiência visual limita a ação da criança em seu ambiente, o aprimoramento de seus atos motores e a sua vivência em atividades interativas com objetos, ou seja, altera o ambiente através do qual o processo de aprendizagem se desenvolve $\mathrm{e}^{(14-16,26)}$.

Segundo Glass, o impacto das alterações visuais pode interferir negativamente no estabelecimento de um contato social eficaz, uma vez que as expressões faciais, os gestos e o contato ocular têm um importante papel na comunicação e na interação social(27). A socialização da criança advém do primeiro grupo social ao qual ela pertence, a família, que possui grande responsabilidade na formação afetiva e também pessoal. Quanto ao comportamento social, a análise estatística mostrou valores mais próximos do limite de significância $p=0,04$. $\mathrm{Na}$ literatura, tem-se mostrado que as crianças portadoras de deficiência visual demonstram dificuldades sociais e emocionais que atingem suas famílias, causando assim impactos em termos de qualidade de vida(28-30). Estudos demonstram a preocupação primária com a socialização, porém, comparando este trabalho com o desenvolvido por Navarro e colaboradores, a questão do comportamento da coordenação aparece de forma mais expressiva ${ }^{(31)}$. A tabela do DNPM normal (demonstrada e utilizada neste estudo) possui validação de uso, pois foi possível observar que os atos comportamentais das crianças pertencentes ao grupo controle, em relação à idade cronológica, foram compatíveis; fato notório durante a coleta de dados. Esse fato demonstra ainda a homogeneidade das escalas avaliadas (motora, de coordenação, de linguagem e social), confirmação referida nos resultados após o teste de Análise de Variância. Desta forma, a tabela pode ser utilizada como método de avaliação de crianças com deficiência visual, visto que outros instrumentos de avaliação precoce para essa faixa etária, que envolvem todos os aspectos conceituais similares aos da tabela aplicada, não estão inclusos na prática clínica ${ }^{(25)}$.

\section{Tabela 1. Resultados obtidos após teste de comparação dos grupos, referente ao desenvolvimento neuropsicomotor}

\begin{tabular}{lcccc}
\hline Comportamento & Motor & Coordenação & Linguagem & Social \\
\hline Sig $(p<0,05)$ & $0,00^{*}$ & $0,00^{*}$ & $0,00^{*}$ & $0,04^{*}$ \\
\hline * = presença de diferença estatística significante & & \\
Aplicação do teste t de Student & & &
\end{tabular}

Tabela 2. Resultados obtidos após teste de comparação dos grupos, referente à funcionalidade visual

\begin{tabular}{lc}
\hline Comportamento & Sig $(\mathbf{p}<\mathbf{0 , 0 5})$ \\
\hline Eficiência visual & $0,00 *$ \\
\hline * $=$ presença de diferença estatística significante & \\
Aplicação do teste t de Student &
\end{tabular}


Anexo 1. Tabela do desenvolvimento neuropsicomotor de Gesell

\begin{tabular}{|c|c|c|c|c|c|c|}
\hline 4 semanas & 12 semanas & 16 semanas & 24 semanas & 28 semanas & 36 semanas & 40 semanas \\
\hline \multicolumn{7}{|c|}{ Motor } \\
\hline $\begin{array}{l}\text { Sem controle } \\
\text { de cabeça }\end{array}$ & $\begin{array}{l}\text { Controle } \\
\text { de cabeça }\end{array}$ & $\begin{array}{l}\text { Postura } \\
\text { simétrica }\end{array}$ & $\begin{array}{l}\text { Controle } \\
\text { detronco }\end{array}$ & $\begin{array}{l}\text { Senta com } \\
\text { apoio }\end{array}$ & Senta & $\begin{array}{l}\text { Senta } \\
\text { sozinho }\end{array}$ \\
\hline Mãos fechadas & $\begin{array}{l}\text { Desaparecimento da } \\
\text { maioria dos reflexos } \\
\text { primitivos }\end{array}$ & $\begin{array}{l}\text { Rolar para os } \\
\text { lados } \\
\text { em bloco }\end{array}$ & $\begin{array}{l}\text { Paraquedas } \\
\text { lateral e } \\
\text { para frente }\end{array}$ & $\begin{array}{l}\text { Equilíbrio } \\
\text { sentado } \\
\text { breve }\end{array}$ & Quatro apoios & Rasteja \\
\hline $\begin{array}{l}\text { Reflexo tônico } \\
\text { cervical assimétrico }\end{array}$ & Criança mais ativa & $\begin{array}{l}\text { Mão na } \\
\text { linha média }\end{array}$ & $\begin{array}{l}\text { Preensão } \\
\text { palmar }\end{array}$ & Leva pé a boca & $\begin{array}{l}\text { Em pé } \\
\text { com apoio }\end{array}$ & $\begin{array}{l}\text { Se mantém em pé } \\
\text { e dá passos com apoio }\end{array}$ \\
\hline $\begin{array}{l}\text { Flexão, abdução } \\
\text { e rotação externa de } \\
\text { membros Inferiores }\end{array}$ & & $\begin{array}{l}\text { Paraquedas } \\
\text { anterior }\end{array}$ & $\begin{array}{l}\text { Rola } \\
\text { independente }\end{array}$ & $\begin{array}{l}\text { Dissociação de } \\
\text { eixos corporais }\end{array}$ & Balança em pé & \\
\hline $\begin{array}{l}\text { Rola parcialmente } \\
\text { para os lados }\end{array}$ & & & $\begin{array}{l}\text { Senta com breve } \\
\text { equilíbrio }\end{array}$ & & Andar de lado & \\
\hline \multicolumn{7}{|c|}{ Coordenação } \\
\hline $\begin{array}{l}\text { Solta objetos } \\
\text { imediatamente }\end{array}$ & $\begin{array}{l}\text { Inicia coordenação } \\
\text { olho-mão }\end{array}$ & $\begin{array}{l}\text { Coordenação olho- } \\
\text { mão e mão-boca }\end{array}$ & $\begin{array}{l}\text { Aproxima } \\
\text { e pega }\end{array}$ & $\begin{array}{l}\text { Balança e bate } \\
\text { brinquedos }\end{array}$ & $\begin{array}{l}\text { Preensão digital } \\
\text { radial }\end{array}$ & $\begin{array}{l}\text { Pinça (polegar e } \\
\text { indicador) }\end{array}$ \\
\hline \multirow[t]{5}{*}{ Mão a boca } & $\begin{array}{l}\text { Puxa } \\
\text { roupa/objetos }\end{array}$ & $\begin{array}{l}\text { Tateia, arranha e } \\
\text { agarra }\end{array}$ & Pega perto & $\begin{array}{l}\text { Aproxima e agarra } \\
\text { com uma das mãos }\end{array}$ & $\begin{array}{l}\text { Deixa cair } \\
\text { e pega }\end{array}$ & $\begin{array}{l}\text { Segura a } \\
\text { mamadeira }\end{array}$ \\
\hline & Braços se agitam & $\begin{array}{l}\text { Mantém } \\
\text { objeto a mão }\end{array}$ & $\begin{array}{l}\text { Mantém } \\
\text { mão livre }\end{array}$ & $\begin{array}{l}\text { Segura } \\
2 \text { objetos }\end{array}$ & $\begin{array}{l}\text { Aproxima } \\
\text { objetos }\end{array}$ & $\begin{array}{l}\text { Resgata objetos } \\
\text { caídos }\end{array}$ \\
\hline & & Pega e olha & & $\begin{array}{l}\text { Descobre peso, } \\
\text { tamanho, forma e } \\
\text { textura }\end{array}$ & & $\begin{array}{l}\text { Empurra, apalpa e } \\
\text { dedilha }\end{array}$ \\
\hline & & & & & & $\begin{array}{l}\text { Noções de } \\
\text { em cima/embaixo, } \\
\text { dentro/fora e } \\
\text { junto/separado }\end{array}$ \\
\hline & & & Linguagem & & & \\
\hline Sem expressão & Arrulha & Arrulha & Rosna e guincha & $\begin{array}{l}\text { Som vocalizado } \\
\text { polissílabo }\end{array}$ & Imita os sons & $\begin{array}{l}\text { Vocalizações como } \\
\text { "da-da", "ma-ma" e } \\
\text { "nha-nha" }\end{array}$ \\
\hline \multirow[t]{2}{*}{ Pequenos ruidos } & Risos & Risos & Resmunga & Som ao chorar & $\begin{array}{l}\text { Compreende o } \\
\text { não }\end{array}$ & \\
\hline & & & & $\begin{array}{l}\text { Gorgoleja e gritos } \\
\text { estridentes }\end{array}$ & & \\
\hline \multicolumn{7}{|c|}{ Social } \\
\hline $\begin{array}{l}\text { Atento ao rosto } \\
\text { humano }\end{array}$ & Resposta facial & Sorri socialmente & $\begin{array}{l}\text { Resposta vocal } \\
\text { espontânea }\end{array}$ & $\begin{array}{l}\text { Olha a própria } \\
\text { imagem e sorri }\end{array}$ & $\begin{array}{l}\text { Segura } \\
\text { mamadeira e } \\
\text { comebiscoito } \\
\text { sozinho }\end{array}$ & $\begin{array}{l}\text { Imita alguns } \\
\text { movimentos } \\
\text { como bater } \\
\text { palmas e dar tchau }\end{array}$ \\
\hline $\begin{array}{l}\text { Percepção de } \\
\text { contorno }\end{array}$ & $\begin{array}{l}\text { Dependente } \\
\text { de fralda }\end{array}$ & $\begin{array}{l}\text { Exige um maior } \\
\text { convívio social }\end{array}$ & $\begin{array}{l}\text { Dependente } \\
\text { de fralda }\end{array}$ & $\begin{array}{l}\text { Interpreta } \\
\text { expressões } \\
\text { faciais }\end{array}$ & $\begin{array}{l}\text { Persistência de } \\
\text { alcance de } \\
\text { objetos distantes }\end{array}$ & $\begin{array}{l}\text { Receio de vozes } \\
\text { desconhecidas }\end{array}$ \\
\hline $\begin{array}{l}\text { Aquieta-se quando } \\
\text { pego no colo }\end{array}$ & & $\begin{array}{l}\text { Dependente } \\
\text { de fralda }\end{array}$ & & $\begin{array}{l}\text { Discriminação de } \\
\text { estranhos }\end{array}$ & $\begin{array}{l}\text { Dependente de } \\
\text { fralda }\end{array}$ & Timidez \\
\hline $\begin{array}{l}\text { Dependente de } \\
\text { fralda }\end{array}$ & & & & $\begin{array}{l}\text { Dependente de } \\
\text { fralda }\end{array}$ & & $\begin{array}{l}\text { Dependente de } \\
\text { fralda }\end{array}$ \\
\hline \multicolumn{7}{|c|}{ Brinquedo } \\
\hline $\begin{array}{l}\text { Luz e brilho, tem } \\
\text { interesse pelas } \\
\text { cores vermelha } \\
\text { e laranja }\end{array}$ & & $\begin{array}{l}\text { Gosta que cantem } \\
\text { e fale com ele, } \\
\text { aprecia briquedos } \\
\text { que estão } \\
\text { ao seu alcance }\end{array}$ & & $\begin{array}{l}\text { Papéis, brinquedos } \\
\text { de borracha macia } \\
\text { que façam } \\
\text { barulho }\end{array}$ & & $\begin{array}{l}\text { Brinquedos que } \\
\text { balancem } \\
\text { (cavalinho de pau) } \\
\text { brincar de esconder } \\
\text { (cobrir o rosto) }\end{array}$ \\
\hline
\end{tabular}


(Continuação) Anexo 1. Tabela do desenvolvimento neuropsicomotor de Gesell

\begin{tabular}{|c|c|c|c|c|c|}
\hline 48 semanas & 60 semanas & 72 semanas & 84 semanas & 96 semanas & 144 semanas \\
\hline \multicolumn{6}{|c|}{ Motor } \\
\hline $\begin{array}{l}\text { Engatinha com } \\
\text { independência }\end{array}$ & $\begin{array}{l}\text { Anda com } \\
\text { independência }\end{array}$ & $\begin{array}{l}\text { Sobe e desce } \\
\text { escadas a sua } \\
\text { maneira }\end{array}$ & $\begin{array}{l}\text { Anda com } \\
\text { rapidez }\end{array}$ & Corre bem & $\begin{array}{l}\text { Apoio unipodal } \\
\text { comequilíbrio } \\
\text { momentâneo }\end{array}$ \\
\hline $\begin{array}{l}\text { Marcha } \\
\text { com apoio }\end{array}$ & Cair súbito & Corre para frente & Corrida em bloco & Anda em círculos & $\begin{array}{l}\text { Marcha em } \\
\text { postura ereta }\end{array}$ \\
\hline $\begin{array}{l}\text { Marcha insegura } \\
\text { sem apoio }\end{array}$ & $\begin{array}{l}\text { Engatinha para } \\
\text { subir escadas }\end{array}$ & Anda de costas & $\begin{array}{l}\text { Agacha-se ao } \\
\text { brincar }\end{array}$ & $\begin{array}{l}\text { Sobe e desce } \\
\text { escadas sozinho }\end{array}$ & Anda em triciclo \\
\hline \multirow[t]{2}{*}{ Ajoelha } & & $\begin{array}{l}\text { Marcha sem } \\
\text { postura ereta }\end{array}$ & $\begin{array}{l}\text { Sobe e desce } \\
\text { com uma mão fixa }\end{array}$ & Pula & $\begin{array}{l}\text { Sobe e desce escadas } \\
\text { usando os } \\
\text { membros inferiores } \\
\text { alternadamente }\end{array}$ \\
\hline & & & & $\begin{array}{l}\text { Marcha com braços } \\
\text { pra fora e para trás }\end{array}$ & $\begin{array}{l}\text { Capacidade de } \\
\text { manobrar }\end{array}$ \\
\hline \multicolumn{6}{|c|}{ Coordenação } \\
\hline $\begin{array}{l}\text { Coloca as mãos } \\
\text { dentro de objetos }\end{array}$ & $\begin{array}{l}\text { Ajuda a virar } \\
\text { uma folha }\end{array}$ & $\begin{array}{l}\text { Arrasta, empurra, } \\
\text { puxa, deixa cair, } \\
\text { segura com rudeza }\end{array}$ & $\begin{array}{l}\text { Rabisco } \\
\text { espontâneo }\end{array}$ & Alinha objetos & $\begin{array}{l}\text { Indicação } \\
\text { de figuras }\end{array}$ \\
\hline $\begin{array}{l}\text { Retira objetos } \\
\text { comfacilidade }\end{array}$ & Segura lápis & $\begin{array}{l}\text { Coloca as coisas } \\
\text { nos lugares }\end{array}$ & & $\begin{array}{l}\text { Imita risco } \\
\text { vertical e circular }\end{array}$ & Dá função a objetos \\
\hline $\begin{array}{l}\text { Segura objetos } \\
\text { pelo cabo }\end{array}$ & Imita rabisco & & & Encaixe quadrado & $\begin{array}{l}\text { Imita riscos } \\
\text { horizontais }\end{array}$ \\
\hline $\begin{array}{l}\text { Dá objetos } \\
\text { e espera } \\
\text { devolução } \\
\text { imediata }\end{array}$ & Encaixe circular & & & Pontapé em bola & $\begin{array}{l}\text { Familiarizada } \\
\text { com } 3 \text { formas: } \\
\text { círculo, quadrado e } \\
\text { triângulo }\end{array}$ \\
\hline Inicia o largar & $\begin{array}{l}\text { Recebe e joga } \\
\text { uma bola }\end{array}$ & & & & $\begin{array}{l}\text { Desabotoa e } \\
\text { abotoa botões }\end{array}$ \\
\hline Inicia o empilhar & Empilha bem & & & & \\
\hline
\end{tabular}

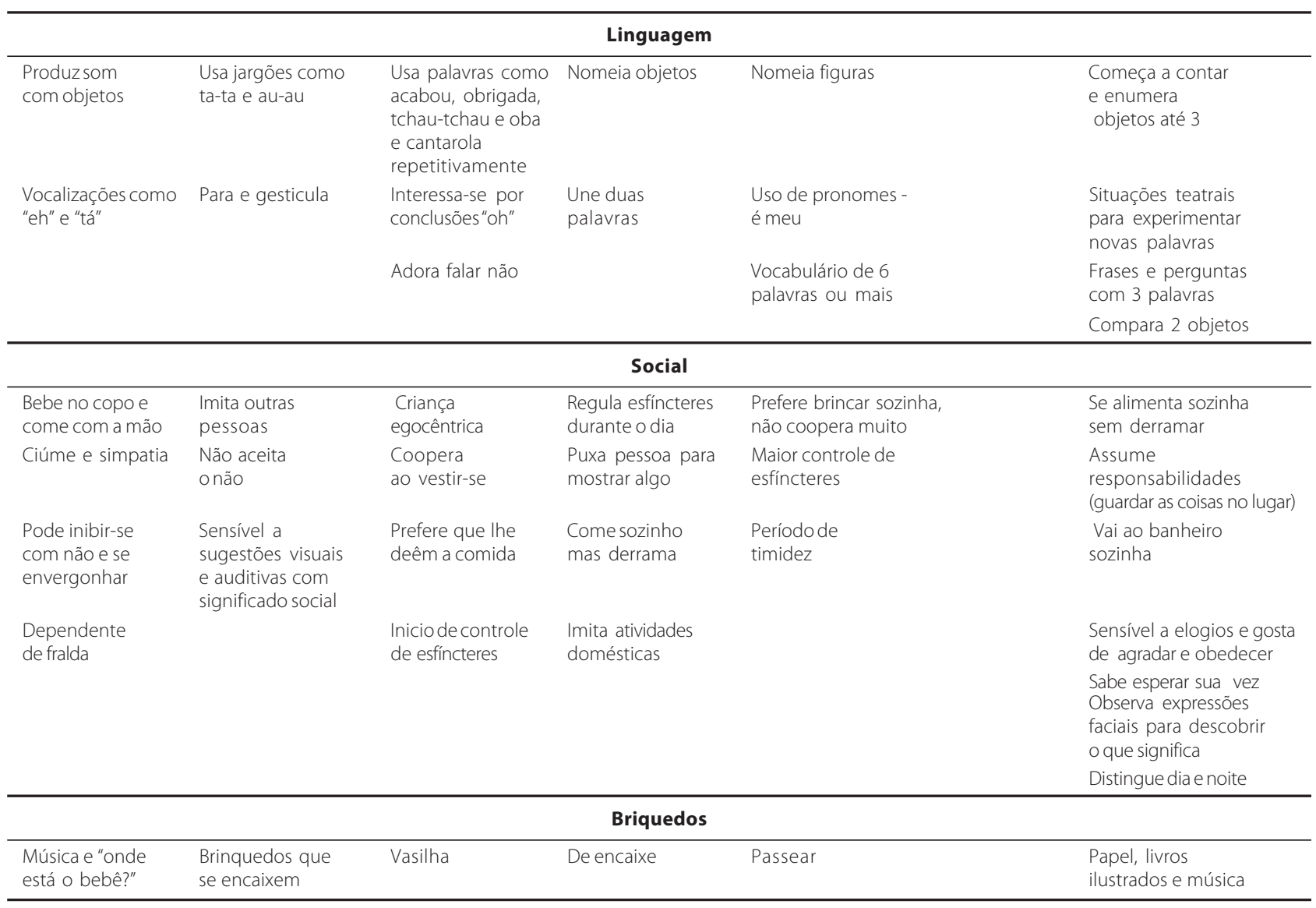


Tabela 3. Medidas dos valores obtidos após comparação dos comportamentos analisados no grupo experimental e controle

\begin{tabular}{llc}
\hline Comportamentos (grupos) & Sig (p<0,05) \\
\hline Motor (experimental) & Coordenação (GE) & 0,89 \\
& Linguagem (GE) & 0,98 \\
& Social (GE) & 1,00 \\
& Média de GC & $0,01^{*}$ \\
Coordenação (experimental)) & Motor (GE) & 0,89 \\
& Linguagem (GE) & 0,99 \\
& Social (GE) & 0,70 \\
& Média de GC & $0,00^{*}$ \\
Linguagem (experimental) & Motor (GE) & 0,98 \\
& Coordenação (GE) & 0,99 \\
& Social (GE) & 0,90 \\
& Média de GC & $0,00^{*}$ \\
& Motor (GE) & 1,00 \\
& Coordenação (GE) & 0,70 \\
& Linguagem (GE) & 0,90 \\
& Média de GC & $0,04^{*}$ \\
\hline
\end{tabular}

* = significância estatística

Aplicação do teste de analise de variância

\section{CONCLUSÃO}

Crianças com deficiência visual apresentam atraso global no desenvolvimento neuropsicomotor e na visão funcional em comparação com as crianças sem esse tipo de deficiência. Referente aos comportamentos abordados na avaliação notou-se, no entanto, que o comportamento da coordenação foi o mais comprometido.

\section{REFERÊNCIAS}

1. Umphred DA. Fisioterapia neurológica. $2^{\underline{a}}$ ed. São Paulo: Manole; 1994.

2. Chak M, Rahi JS; British Congenital Cataract Interest Group. The health-related quality of life of children with congenital cataract: findings of the British Congenital Cataract Study. Br J Ophtahlmol. 2007;91 (7):922-6.

3. Santos LC, Passos JEOS, Rezende ALG. Os efeitos da aprendizagem psicomotora no controle das atividades de locomoção sobre obstáculos em crianças com deficiência da visão. Rev Bras Educ Espec. 2007;13(3):365-80.

4. Castro EM. Desenvolvimento sensório-motor e perceptivo-motor de populações especiais [tese]. São Paulo: Universidade Estadual Paulista; 2006

5. Figueira MMA. Assistência fisioterapia à criança portadora de cegueira congênita. Rev Benjamin Constant. 2000;6(17):10-23.

6. Masini EFS. A educação do portador de deficiência visual: as perspectivas do vidente e do não vidente. In: Brasil. Ministério da Educação e do Desporto. Secretaria de Educação Especial. Tendências e desafios da deficiência visual. Brasília: MEC/SEESP; 1994. p.82-103.

7. Gesell A. A criança do 0 aos 5 anos. São Paulo: Martins Fontes; 1999.

8. Graziano RM, Leone CR. Problemas oftalmológicos mais frequentes e desenvolvimento visual do pré-termo extremo. J Pediatr (Rio J). 2005;81 (1 Supl):S95-100.
9. Resnikoff S, Pascolini D, Etya'a1e D, Kocur I, Pararajasegaram R, Pokharel GP, Mariotti SP. Global data on visual impairment in the year 2002. Bull World Health Organ. 2004;82(11):844-51

10. Organização Mundial de Saúde. O atendimento de crianças com baixa visão. Programa para a prevenção de cegueira da OMS. Bangkok: OMS;1994. p.32-9.

11. Gilbert C, Foster A. Childhood blindness in the context of VISION 2020-the right to sight. Bull World Health Organ. 2001;79(3):227-32.

12. Agbeja AM, Cookey-Gam Al. Rehabilitation of the blind: a review. East Afr Med J. 1992;69(6):341-4. Review.

13. Ramírez F. Intervenção educativa. Caidv. Espanã, 1989: 112-3.

14. Backin JS. Your child's development. In: Geralis E, editor. Children with cerebral palsy: A parent's guide. Bethesda: Woodbine House: 1992. p.175-208.

15. Lamonica DAC. Linguagem na paralisia cerebral. In: Ferreira LP, Befi-Lopes DM Limongi SCO, organizadores. Tratado de fonoaudiologia. São Paulo: Roca; 2004 p.967-76.

16. Miller G, Clark GD. Paralisias cerebrais: causas, consequências e condutas. São Paulo: Manole; 2002.

17. American Foundation for the Blind [Internet]. United States American: ABF. [cited from: Erin JN, Paul B. Functional vision assessment and instruction of children and youths in academic programs. In: Corn AL, Koenig AJ, editors. Foundations of low vision: Clinical and functional perspectives. New York: AFB Press; 1966. p.185-220. Available from: http://www.iowa.gov/educate.

18. Childhood cataract: magnetude, management, economics and impact. Community Eye Health. 2004;17(50):17-8.

19. Santana A, Koller K, Waiswol M. Anoftalmia associada à catarata congênita: relato de caso. Arq Bras Oftalmol. 2005;68(3):385-8.

20. Zetterström C, Lundvall A, Kugelberg M. Cataracts in children. J Cataract Refract Surg. 2005;31(4):824-40. Review.

21. Cruz CB, Endriss D, Ventura B, Ventura L. Catarata na infância: perfil socioeconômico, gestacional e desenvolvimento neuropsicomotor. Arq Bras Oftalmol. 2005;68(1):9-13.

22. Amaral ACT, Tabaquim MLM, Lamônica DAC. Avaliação das habilidades cognitivas, da comunicação e neuromotoras de crianças com risco de alterações do desenvolvimento. Rev Bras Educ Espec. 2005;11(2):185-200

23. Preda C. Test of visual-motor integration: construct validity in a comparison with the Beery-Buktenica Developmental Test of Visual-Motor Integration. Percept Mot Skills. 1997;84(3 Pt 2):1439-43.

24. Cioni G, Bertuccelli B, Boldrini A, Canapicchi R, Fazzi B, Guzzetta A, Mercuri E. Correlation between visual function, neurodevelopmental outcome, and magnetic resonance imaging findings in infants with periventricular leucomalacia. Arch Dis Child Fetal Neonatal. 2000;82(2):F134-40.

25. Dale N, Salt A. Early support developmental journal for children with visual impairment: the case for a new developmental framework for early intervention. Child Care Health Dev. 2007;33(6):684-90.

26. Haddad MA, Lobato FJ, Sampaio MW, Kara-José N. Pediatric and adolescent population with visual impairment: study of 385 cases. Clinics (Sao Paulo). 2006; 61(3):239-46.

27. Glass P. Development of the visual system and implications for early intervention. Infants Young Child. 2002;15(1):1-10.

28. Tartarella MB, Kawakami LT, Scarpi MJ, Hayashi S. Aspectos cirúrgicos em catarata congênita. Arq Bras Oftalmol. 1995;58(1):24-8.

29. Wolffsohn JS, Cochrane AL. Design of the low vision quality-of-life questionnaire (LVQOL) and measuring the outcome of low-vision rehabilitation. Am J Ophthalmol. 2000;130(6):793-802.

30. Boulton M, Haines L, Smyth D, Fielder A. Health-related quality of life of children with vision impairment or blindness. Dev Med Child Neurol. 2006;48(8):656-61.

31. Navarro AS, Fukujima MM, Fontes SV, Matas SLA, Prado GF. Balance and motor coordination are not fully developed in 7 years old blind children. Arq Neuropsiquiatr. 2004;62(3A):654-7. 